\title{
Combination of Limosilactobacillus fermentum MG4231 and MG4244 attenuates lipid accumulation in high-fat diet-fed obese mice
}

\author{
S.J. Kim ${ }^{1}$, S.-I. Choi ${ }^{* \# \#}$, M. Jang ${ }^{2}$, Y.-A. Jeong ${ }^{3}$, C.-H. Kang ${ }^{3}$ and G.-H. Kim $^{4 * \#}$ \\ ${ }^{1}$ Department of Health Functional Materials, Duksung Women's University, 144 gil, Dobong-gu, Seoul, 01369, Republic \\ of Korea; ${ }^{2}$ Department of Food and Life Science, Inje University, Gimhae, Republic of Korea; ${ }^{3}$ RED Center, MEDIOGEN \\ Co., Ltd., Seoul, Republic of Korea; ${ }^{4}$ Department of Food and Nutrition, Duksung Women's University, 33, Samyang-ro \\ 144-gil, Dobong-gu, Seoul, 01369, Republic of Korea; langdeveu74@duksung.ac.kr; ghkim@duksung.ac.kr; "these authors \\ contributed equally to this work
}

Received: 14 December 2020 / Accepted: 17 April 2021

(c) 2021 Wageningen Academic Publishers

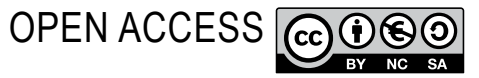

RESEARCH ARTICLE

\begin{abstract}
We investigated the anti-obesity effect and the underlying mechanisms of action of human-derived Limosilactobacillus fermentum MG4231, MG4244, and their combination, in high-fat diet-induced obese mice. Administration of the Limosilactobacillus strains decreased body weight gain, liver and adipose tissue weight, and glucose tolerance. Serum levels of total cholesterol, low-density lipoprotein-cholesterol, and leptin were reduced, while adiponectin increased. The administration of Limosilactobacillus strains improved the histopathological features of liver tissue, such as hepatic atrophy and inflammatory penetration, and significantly reduced the content of triglyceride in the liver. Limosilactobacillus administration discovered a significant reduction in the size of the adipocytes in the epididymal tissue. Limosilactobacillus treatment significantly reduced the expression of important regulators in lipid metabolism, including peroxisome proliferator-activated receptor $\gamma$, CCAAT/enhancer-binding protein $\alpha$, fatty acid synthase (FAS), adipocyte-protein 2, and lipoprotein lipase in the epididymal tissue. Also, Limosilactobacillus lowered sterol regulatory element-binding protein 1-c and FAS in the liver tissue. Such changes in the expression of these regulators in both liver and epididymis tissue were caused by Limosilactobacillus upregulating phosphorylation of AMP-activated protein kinase and acetyl-CoA carboxylase. Therefore, we suggest that the use of the combination of L. fermentum MG4231 and MG4244, as probiotics could effectively inhibit adipogenesis and lipogenesis from preventing obesity.
\end{abstract}

Keywords: high-fat diet, L. fermentum, obesity, AMPK, probiotics

\section{Introduction}

Obesity is defined as excessive body fat accumulation owing chronic energy intake and lack of exercise and a body mass index (BMI) greater than or equal to 30 (WHO, 2020). The proportion of overweight or obese people continues to rise and is a serious problem worldwide (Nuttall, 2015). Obesity is a complex, multifactorial disease primarily attributed to genetic risk factors and is recognised as an important cause of metabolic disorders, such as insulin resistance, diabetes, hyperlipidemia, hypertension, cardiovascular disease, and non-alcoholic fatty liver disease (NAFLD) (Paley and Johnson, 2018).
Probiotics are living, non-pathogenic microorganisms whose symbiosis with intestinal microbes has a beneficial effect on the health of the host. Limosilactobacillus and Bifidobacterium are currently the most commonly used lactic acid bacteria (LAB) as probiotics, which mainly improve gastrointestinal disorders and inhibit excessive growth of pathogenic intestinal bacteria (Wilkins and Sequoia, 2017). Probiotics must satisfy a variety of conditions, such as safety, antibiotic susceptibility, resistance to digestive enzymes or gastric acid, adhesion to epithelial cells in the gut, and functional properties. In principle, they should be commercially available as probiotics (Bubnov et al., 2018). 
Recently, with the increasing interest in intestinal microorganisms, results regarding the relevance and usefulness of probiotics in the treatment of various diseases by improving immunity, reducing blood glucose levels, antioxidant, and anti-cancer activities have been continuously presented (Tarrah et al., 2018; Zhang et al., 2015). Several studies have reported that obesity is related to structural and functional changes of LAB in gut microbiota (Abenavoli et al., 2019; Choi and Chang, 2015). Lacticaseibacillus (formerly Lactobacillus) rhamnosus hsryfm 1301 improved lipid metabolism by regulating the gut microbiota in hyperlipidemic rats (Chen et al., 2014). Lactiplantibacillus (formerly Lactobacillus) plantarum FRT10 alleviated high fat diet (HFD)-induced obesity in mice by regulating the peroxisome proliferator-activated receptor (PPAR) $\alpha$ signalling pathway and gut microbiota (Cai et al., 2020). Latilactobacillus (formerly Lactobacillus) sakei CJLS03 alleviated body weight increase, short-chain fatty acids (SCFAs), the gut microbiota and various obesity-associated biomarkers (Ji et al., 2019). Differences in the ratio of Firmicutes/Bacteroidetes lead to obesity by increasing energy production and metabolism (Ley et al., 2005). Some probiotics have been found to elicit anti-obesity activity by regulating lipid and glucose metabolism. $L$. plantarum LMT1-48 showed antiobesity effects, including body weight loss, reduction in abdominal fat volume, and decrease in leptin and total cholesterol levels in Enterobacter cloacae-induced HFD-fed obese mice (Choi et al., 2019). L. plantarum Ln 4 attenuated obesity and type- 2 diabetes biomarkers in HFD-induced mice (Lee et al., 2018).

In our previous study, two human-derived strains of Limosilactobacillus (formerly Lactobacillus) fermentum MG4231 and MG4244 were isolated. These strains have been shown to inhibit adipocyte differentiation in vitro and have been identified as probiotics candidates (Kang et al., 2018; Kim et al., 2020). Therefore, this study aimed to evaluate the anti-obesity activity of $L$. fermentum MG4231, MG4244, and their combination to identify the underlying molecular mechanisms associated with lipid metabolism in a HFD-induced obese mouse model.

\section{Materials and methods}

\section{Materials}

Antibodies against peroxisome proliferator-activated receptor gamma (PPAR $\gamma$ ), CCAAT/enhancer-binding protein alpha (C/EBP $\alpha)$, fatty acid synthase (FAS), adipocyte-protein 2 (aP2), lipoprotein lipase (LPL), sterol regulatory element binding protein 1-c (SREBP-1c), acetylCoA carboxylase (ACC), phosphorylated ACC (p-ACC), AMP-activated protein kinase (AMPK), phosphorylated AMPK (p-AMPK), and $\beta$-actin were purchased from
Mybiosource (San Diego, CA, USA). All other reagents were purchased from Sigma-Aldrich (St. Louis, MO, USA).

\section{Preparation of Limosilactobacillus fermentum MG4231 and MG4244}

The L. fermentum MG4231 and MG4244 powders used in this study were supplied by Mediogen Co., Ltd. (Jecheon, Korea). Each strain was activated by culturing in a general medium. To prepare Limosilactobacillus powder, freshly harvested bacterial pellets were mixed well with the cryoprotectant mixture (Nguyen et al., 2020). The bacterial cells were dispersed in wall materials and dried (Heto Drywinner, Allerod, Denmark). The powdered cells were harvested and collected in polythene bags, wrapped in aluminium foil, and stored at $4{ }^{\circ} \mathrm{C}$ until further use.

\section{Animals and experimental design}

Male C57BL/6J mice (4-wk old, 18-20 g) were obtained from Orient-Bio Inc. (Seongnam, Korea). Mice were housed in an air-conditioned room at $23 \pm 1.0^{\circ} \mathrm{C}$ and $45 \pm 5 \% \mathrm{RH}$, with a light and dark cycle of $12 / 12 \mathrm{~h}$. Mice were fed at random with standard laboratory chow and water ad libitum. All mice were treated in accordance with the Guide for the Care and Use of Laboratory Animals (NRC, 1996). Experimental protocols were approved by the Institutional Animal Care and Use Committee (IACUC) of Duksung Women's University (Approval no. 2019-003-010), and every effort was made to minimise suffering.

To induction of obesity, mice were randomly distributed into the following five groups ( $\mathrm{n}=6 /$ group): normal diet, HFD, HFD + L. fermentum MG4231, HFD + L. fermentum MG4244, and HFD + the combination of MG4231 and MG4244 (at a mass ratio of 1:1). MG4231, MG4244, or their $1: 1$ combination $\left(2 \times 10^{8} \mathrm{cfu} /\right.$ mouse $)$ was dissolved in sterile phosphate-buffered saline (PBS) and administered orally once daily for 8 weeks with HFD supplement. The HFD group were fed a diet containing $60 \%$ fat, $20 \%$ protein, and 20\% carbohydrate (D12492, Research Diets Inc., New Brunswick, NJ, USA). ND group received a diet containing $14 \%$ fat, $21 \%$ protein, and $64 \%$ carbohydrate (Furina Inc., Seongnam, Korea). Dosages of these strains were established based on results obtained from in vitro preliminary studies (Kim et al., 2020) and those used in previous studies (Hussain et al., 2020; Li et al., 2020).

\section{Body weight and food intake}

Mice were monitored daily. Body weight and food intake were measured weekly. Food was supplied to mice simultaneously, and the amount of food consumed was calculated from the amount that remained in the cage. 


\section{Oral glucose tolerance test}

Oral glucose tolerance test (OGTT) was performed after the 8-week treatment period. Following fasting for more than $12 \mathrm{~h}$, glucose $(2 \mathrm{~g} / \mathrm{kg}$ b.w.) was orally administered to the mice. Thereafter, blood glucose levels were measured at 30,60, 90, and $120 \mathrm{~min}$ by tail vein puncture using a glucose meter (Glucocard II; Arkray Inc., Kyoto, Japan) (Andrikopoulos et al., 2008).

\section{Weight of liver and adipose tissues}

Following a $12 \mathrm{~h}$ fast, at the end of the experiment, all groups of mice were anesthetized by $\mathrm{CO}_{2}$ inhalation and euthanised by cardiac puncture. The liver and adipose tissue (epididymal, intestinal, retroperitoneal, perirenal, and subcutaneous) were extracted, rinsed with PBS buffer, weighed, and visually inspected. All tissue samples were frozen in liquid nitrogen and stored at $-70^{\circ} \mathrm{C}$ until analysis.

\section{Serum biochemical analysis}

Blood samples were collected in EDTA-coated tube, placed at room temperature for a minimum of $30 \mathrm{~min}$, and centrifuged at 3,000 rpm for $20 \mathrm{~min}$ at $4{ }^{\circ} \mathrm{C}$. Serum levels of alanine amino transferase (ALT), aspartate aminotransferase (AST), triglyceride (TG), total cholesterol (TC), high-density lipoprotein cholesterol (HDL), low-density lipoprotein cholesterol (LDL), and glucose were measured using a chemistry analyser (Beckman Coulter, Inc. Brea, CA, USA). Plasma leptin or adiponectin concentrations were enzymatically quantified using a commercial ELISA kit (Enzo-Diagnostic, Farmingdale, NY, USA).

\section{Histopathological examination}

We analysed the histopathological alterations in the liver and epididymal tissues were analysed to assess the effect of the Limosilactobacillus strains. Tissues were fixed in 10\% formalin for $24 \mathrm{~h}$, embedded in paraffin, sectioned at $4 \mu \mathrm{m}$ in liver tissue and $8 \mu \mathrm{m}$ in adipose tissue, and stained with haematoxylin and eosin (H\&E). Slides were subjected to microscopic examination. Histopathological changes in liver tissues were quantified and expressed by the summation of individual 6-grades (score 0 to 5) for hepatic steatosis, inflammation, and hepatocellular hypertrophy as follows: $0=$ normal 1 = minimal $; 2$ slight $; 3=$ moderate $4=$ marked; and 5 = severe, numerically evaluated according to semi-quantitative pathological criteria (Liang et al., 2014). In addition, all slides of epididymal adipose tissue were prepared by randomly selecting from three different parts, and the diameter of 30 adipocytes was measured using an image analyser (Tomoro Scope Eye 3.6, Techsan, Seoul, Korea) equipped with a camera (300MI CMOS, Artray Co., Ltd., Tokyo, Japan). All histological evaluation procedures were blindly performed by a board-certified toxicologic pathologist.

\section{Liver triglycerides analysis}

The liver tissue (100 mg) was homogenised by adding $1 \mathrm{ml}$ of $5 \%$ Nonidet-P40 substitute. The homogenate was heated in a water bath $\left(80-100{ }^{\circ} \mathrm{C}\right)$ for $5 \mathrm{~min}$ and then cooled to room temperature, after which the heating was repeated one more time to solubilise all TG. The homogenate was centrifuged at 5,000 rpm for $2 \mathrm{~min}$ to remove insoluble materials, and the supernatant was diluted 10 times before analysis (Srivastava et al., 2006). The TG content in the lipid extract was measured using a TG quantification kit (Sigma-Aldrich). The measured value was quantified by the protein concentration of the lipid extract measured by Bradford analysis.

\section{Western blot analysis}

The levels of lipogenic protein expression in liver and adipose tissue were determined by western blot analysis. Briefly, tissues were lysed by RIPA buffer containing a protease inhibitor cocktail (Roche, Nutley, NJ, USA) and centrifuged at $13,000 \mathrm{rpm}$ for $20 \mathrm{~min}$ at $4{ }^{\circ} \mathrm{C}$. Protein levels in lysates were quantified using the bicinchoninic acid assay (Walker, 1994). The protein $(20 \mu \mathrm{g})$ was mixed with $10 \%$ sodium-dodecyl sulphate (SDS) loading buffer, electrophoresed on a 10\% SDS-polyacrylamide gel, transferred to a polyvinylidene difluoride (PVDF) membrane, and blocked for 90 min with 5\% skim milk in Tris-buffered saline with Tween (TBST). Membranes were incubated overnight with primary antibodies against PPAR $\gamma$, SREBP-1c, FAS, aP2, LPL, p-ACC, ACC, p-AMPK, and AMPK $(1: 1000)$ or $\beta$-actin $(1: 3,000)$ in $5 \%$ skim milk at $4{ }^{\circ} \mathrm{C}$, and washed multiple times with TBST buffer. Membranes were further reacted with goat anti-rabbit IgGHRP conjugated secondary antibody at room temperature for 90 min and washed multiple times with TBST buffer. Proteins were visualised using the ECL detection kit (Thermo-Fisher Scientific Inc., Cambridge, MA, USA) and quantified using the Image J program (National Institute of Health, Bethesda, MD, USA). The expression level relative to $\beta$-actin was subsequently determined.

\section{Statistical analysis}

The results of the experiments performed in triplicate are presented as the mean \pm standard deviation. Statistical analysis was conducted using one-way analysis of variance (ANOVA) using SPSS Version 22 (IBM Corp., Armonk, NY, USA). Significant differences between groups were analysed using Duncan's multiple range tests. Statistical significance was accepted for $P$ values $<0.05$. The relation between bacterial community and experimental factors was 
performed by statistical analysis in the R Software V.4.0.2 (R Development Core Team, 2020).

\section{Results}

\section{Effect of Limosilactobacillus fermentum combination on change of body weight and food intake}

Changes in body weight and food intake were observed in obese mice fed a HFD for 8-weeks (Figure 1). The weight gain of the HFD group $(19.62 \pm 1.99 \mathrm{~g})$ increased significantly by approximately 3.8 -fold compared to the ND group $(5.18 \pm 1.45 \mathrm{~g})$, and it was confirmed that obesity was effectively induced. However, in compared to the HFD-fed mice, the MG4244 $(16.31 \pm 1.92 \mathrm{~g})$ and the combination $(14.18 \pm 2.82 \mathrm{~g})$ groups exhibited a reduced weight gain without a reduction in dietary intake.

\section{Effect of Limosilactobacillus fermentum combination on OGTT}

Changes in glucose tolerance in HFD-fed mice were measured by OGTT following induction completion. As shown in Figure 2A, blood glucose levels in all groups increased markedly $30 \mathrm{~min}$ after glucose administration and recovered to an almost fasting level at $120 \mathrm{~min}$. However, blood glucose levels $60 \mathrm{~min}$ post-administration were significantly reduced in the combination-treated group $(270.0 \pm 20.28 \mathrm{mg} / \mathrm{dl})$ compared to those in the HFD group $(332.30 \pm 32.10 \mathrm{mg} / \mathrm{dl})$. Figure $2 \mathrm{~B}$ shows the area under the curve (AUC) calculated for all groups to express glucose excursion. The AUC of the HFD group was 1,142 \pm 98.40 $\mathrm{mg} / \mathrm{dl}$, which was two times higher than that of the $\mathrm{ND}(693.9 \pm 70.26 \mathrm{mg} / \mathrm{dl})$ group. In contrast, that of the combination-treated group was significantly lower than that of the HFD group at $1,005 \pm 98.40 \mathrm{mg} / \mathrm{dl}$.

\section{Effect of Limosilactobacillus fermentum combination on liver and adipose tissue weight}

Tissue weight was measured to determine the extent of fat accumulation in the liver and adipose tissue after 8 weeks of HFD-feeding, and the results are shown in Table 1. The liver weight of the HFD group was increased by 0.3 g compared to the ND group. Alternatively, the liver tissue weight of the combination-treated group was significantly lower than that of the HFD group. Epididymal adipose tissue is white fat, as a major indicator of visceral obesity in mice, it causes metabolic changes, such as blood lipid content (Trayhurn and Beattie, 2001). The epididymal and subcutaneous adipose tissue weight of the HFD group increased 8- and 13-fold, respectively, compared to the ND group. On the other hand, these tissue weights were significantly decreased in all L. fermentum-treated groups. In particular, in the combination-treated group, epididymal and subcutaneous tissue weights were reduced by 22 and $58 \%$, respectively. Total adipose tissue weight extracted, excluding liver weight, was significantly reduced by $39 \%$ in the combination-treated group compared to the HFD group.

\section{Effect of Limosilactobacillus fermentum combination on serum level}

Eight weeks after HFD supply, the biochemical changes of serum in HFD-fed mice were confirmed (Table 2). ALT, AST, TC, and LDL levels of the HFD group were significantly higher than that of the ND group. However,
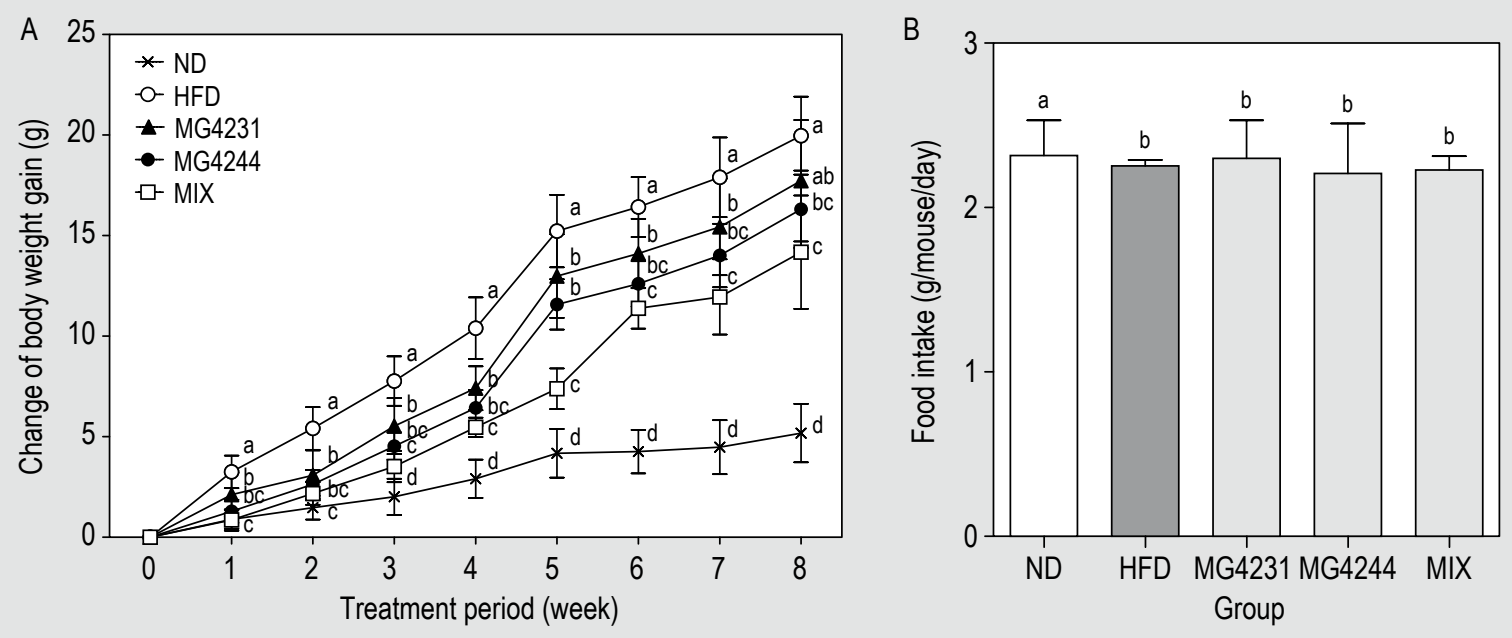

Figure 1. Effect of Limosilactobacillus fermentum strains on (A) body weight gain and (B) food intake in HFD-induced obese mice. Body weight and food intake were measured weekly. ND = normal-diet-fed, HFD = high-fat-diet-fed, MIX = combination of L. fermentum MG4231 and MG4244 in a 1:1 ratio. Results are presented as the mean \pm standard deviation ( $n=6)$. Different letters indicate significant differences between means at $P<0.05$. 

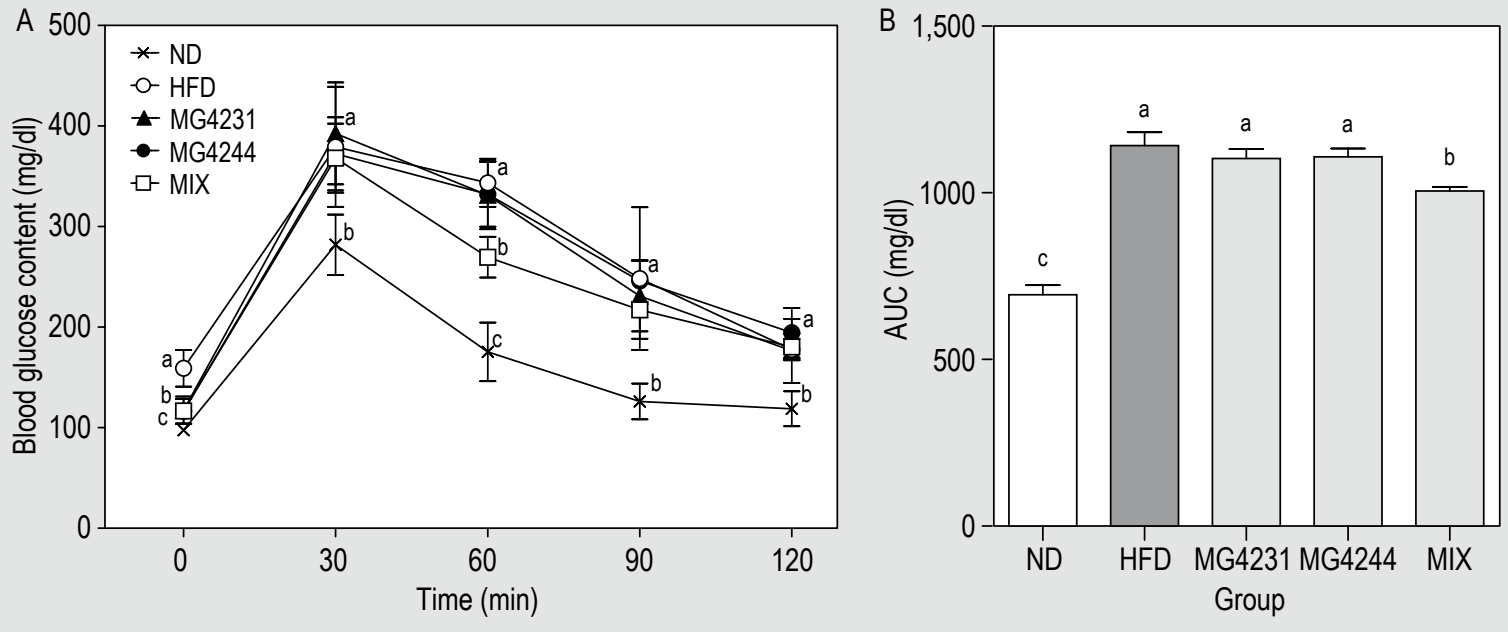

Figure 2. Effect of Limosilactobacillus fermentum strains on glucose tolerance in HFD-induced obese mice. (A) Blood glucose concentration and (B) area under the curve (AUC). ND = normal-diet-fed, HFD = high-fat-diet-fed, MIX = combination of $L$. fermentum MG4231 and MG4244 in a 1:1 ratio. Glucose tolerance was determined by measuring blood glucose levels using the oral glucose tolerance test (OGTT) after the 8-week treatment period. Results are presented as the mean \pm standard deviation $(n=6)$. Different letters indicate significant differences between means at $P<0.05$.

Table 1. Weight of liver and adipose tissues in high fat diet (HFD)-induced obese mice. ${ }^{1}$

\begin{tabular}{|c|c|c|c|c|}
\hline \multirow[t]{2}{*}{ Groups $^{2}$} & \multicolumn{3}{|c|}{ Adipose tissue weight (g) } & \multirow[t]{2}{*}{ Total adipose tissue weight ( $\mathrm{g}$ ) } \\
\hline & Liver & Epididymal & Subcutaneous & \\
\hline ND & $1.00 \pm 0.09^{c}$ & $0.32 \pm 0.06^{c}$ & $0.20 \pm 0.07^{c}$ & $0.91 \pm 0.19^{c}$ \\
\hline HFD & $1.29 \pm 0.17^{a}$ & $2.53 \pm 0.28^{a}$ & $2.62 \pm 0.19^{a}$ & $7.87 \pm 0.57^{\mathrm{a}}$ \\
\hline HFD + MG4231 & $1.14 \pm 0.15^{\mathrm{ab}}$ & $2.01 \pm 0.39^{b}$ & $1.49 \pm 053^{b}$ & $5.33 \pm 1.59^{b}$ \\
\hline HFD + MG4244 & $1.15 \pm 0.05^{\mathrm{ab}}$ & $2.13 \pm 0.10^{\mathrm{ab}}$ & $1.29 \pm 0.19^{b}$ & $5.22 \pm 0.50^{b}$ \\
\hline Combination & $1.12 \pm 0.08^{b}$ & $1.95 \pm 0.55^{b}$ & $1.10 \pm 0.39^{b}$ & $4.81 \pm 1.45^{b}$ \\
\hline
\end{tabular}

ALT levels of MG4231, MG4244 or combination-treated group were considerably lower than those of the HFD group, and TC and LDL levels were significantly lower in L. fermentum-treated groups. TG, glucose, and HDL increased in all groups fed HFD but did not decrease due to the administration of Limosilactobacillus strains. Leptin levels increased approximately 11-fold in the HFD group compared to the ND group. In contrast, leptin levels were reduced by 24 and $10 \%$ in the MG4231 and MG4244 groups, respectively, compared to the HFD group. In particular, the combination-treated group showed the most significant decrease by $41.1 \%$. Adiponectin levels were significantly lower in the HFD group than in the ND group, whereas the adiponectin levels reduced by HFD feeding recovered to levels similar to those of the ND group in the combinationtreated groups.

\section{Effect of Limosilactobacillus fermentum combination on histological change of liver and adipose tissue}

The effects of L. fermentum alone or a combination of strains on steatosis and lipogenesis were evaluated by analysing histopathological changes in liver and adipose tissues. Fat accumulation in the cytoplasm of hepatocytes is observed in the form of a clear vacuolar (Figure 3A). The size of these vacuoles depends on the progression of liver damage and the ratio of TG to phospholipid in accumulated fat (Sethunath et al., 2018). The liver tissue 
Table 2. Biochemical analysis of plasma in high fat diet (HFD)-induced obese mice. ${ }^{1,2}$

\begin{tabular}{|c|c|c|c|c|c|}
\hline \multirow[t]{2}{*}{ Profile } & \multicolumn{5}{|l|}{ Group } \\
\hline & ND & HFD & MG4231 & MG4244 & Combination \\
\hline AST (IU/I) & $58 \pm 5.0^{b}$ & $114 \pm 24.0^{\mathrm{a}}$ & $101 \pm 10.0^{\mathrm{a}}$ & $95 \pm 16.0^{a}$ & $96 \pm 15.0^{a}$ \\
\hline ALT (IU/I) & $24 \pm 2.0^{c}$ & $93 \pm 28.0^{\mathrm{a}}$ & $68 \pm 14.0^{b}$ & $50 \pm 11.0^{\mathrm{b}}$ & $53 \pm 17.0^{b}$ \\
\hline TG (mg/dl) & $19.3 \pm 2.6^{\mathrm{a}}$ & $29.1 \pm 2.3^{b}$ & $29.7 \pm 4.7^{b}$ & $29.5 \pm 6.8^{b}$ & $28.5 \pm 4.0^{b}$ \\
\hline TC (mg/dl) & $84 \pm 7.0^{c}$ & $156 \pm 10.0^{\mathrm{a}}$ & $136 \pm 27.0^{b}$ & $143 \pm 13.0^{\mathrm{ab}}$ & $130 \pm 25.0^{b}$ \\
\hline $\mathrm{HDL}(\mathrm{mg} / \mathrm{dl})$ & $46.7 \pm 3.0^{\mathrm{b}}$ & $60.2 \pm 3.2^{\mathrm{a}}$ & $57.2 \pm 4.0^{\mathrm{a}}$ & $58.3 \pm 5.3^{a}$ & $57.8 \pm 5.3^{\mathrm{a}}$ \\
\hline LDL (mg/dl) & $4.9 \pm 1.8^{b}$ & $7.3 \pm 0.9^{a}$ & $5.5 \pm 2.2^{b}$ & $5.6 \pm 1.0^{b}$ & $5.2 \pm 1.6^{b}$ \\
\hline Glucose (mg/dl) & $233.3 \pm 39.2^{b}$ & $293.2 \pm 14.1^{\mathrm{a}}$ & $293.9 \pm 37.1^{a}$ & $299.0 \pm 21.6^{a}$ & $300.5 \pm 28.1^{a}$ \\
\hline Leptin (ng/ml) & $4.4 \pm 0.8^{d}$ & $48.7 \pm 2.51^{\mathrm{a}}$ & $36.5 \pm 3.6^{b}$ & $42.9 \pm 5.92^{\mathrm{ab}}$ & $28.7 \pm 5.4^{c}$ \\
\hline Adiponectin ( $\mu \mathrm{g} / \mathrm{ml})$ & $109.4 \pm 13.3^{\mathrm{a}}$ & $89.9 \pm 6.4^{c}$ & $91.92 \pm 9.9 \mathrm{bc}$ & $102.3 \pm 12.5^{b c}$ & $107.7 \pm 10.5^{\mathrm{a}}$ \\
\hline
\end{tabular}

${ }^{1}$ Results are presented as mean \pm standard deviation $(n=6)$. Different letters indicate significant difference between means at $P<0.05$ by Duncan's multiple range test.

${ }^{2} \mathrm{ND}=$ normal-diet fed; HFD = high-fat-diet fed; combination: (HFD +) Limosilactobacillus fermentum MG4231 and MG4244 in a 1:1 ratio.

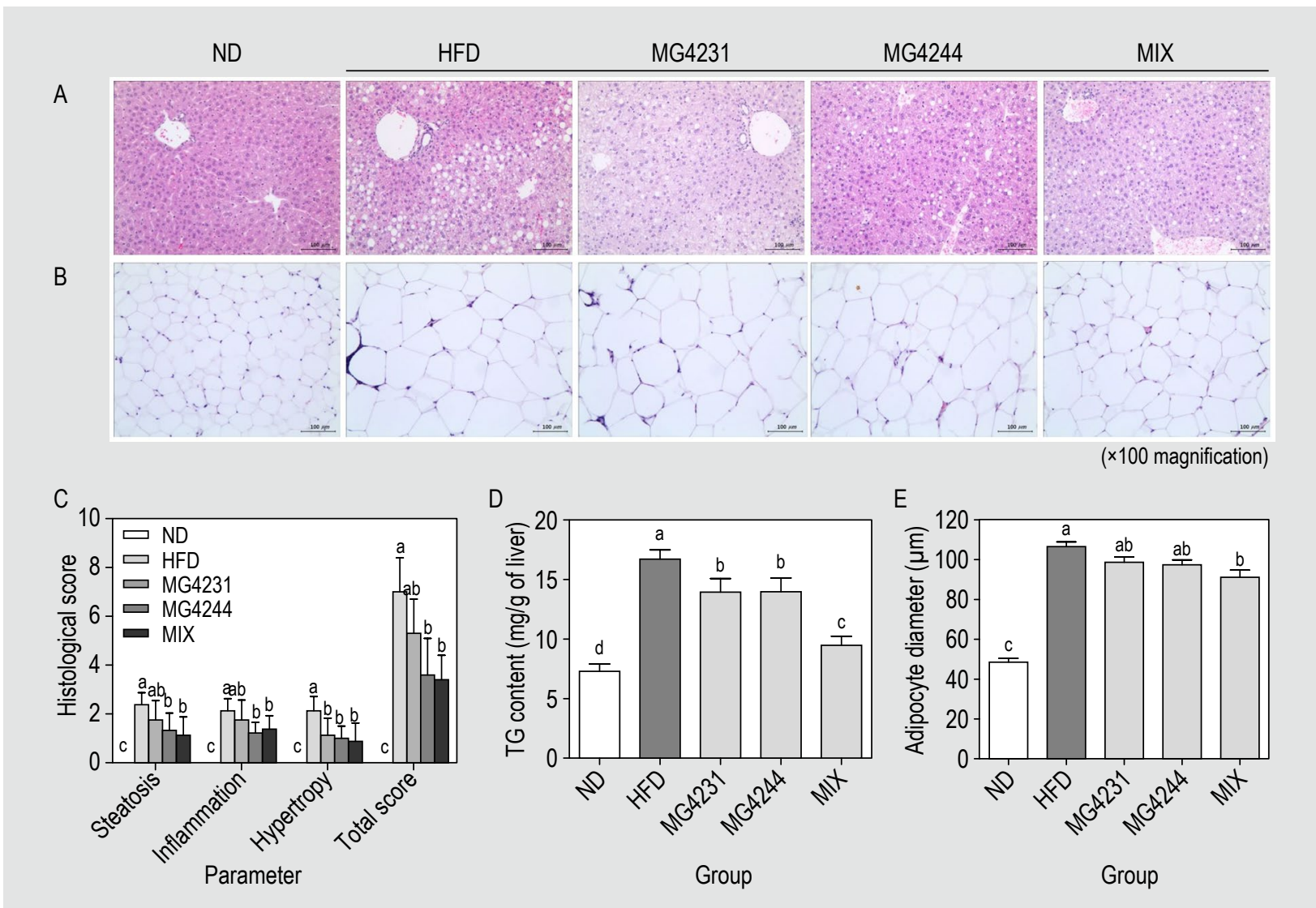

Figure 3. Histological change in liver and epididymal adipose tissue in HFD-induced obese mice. Representative photographs of H\&E staining (original magnification $\times 100$ ) of (A) liver, (B) epididymal tissues, (C) liver histopathological score, (D) liver TG contents, and $(E)$ adipocyte diameter. The diameter of 30 adipocytes was measured using an image analyser. Results are presented as the mean \pm standard deviation $(\mathrm{n}=6)$. Different letters indicate significant differences between means at $P<0.05$. ND $=$ normal-diet fed; HFD = high-fat-diet fed; MIX: (HFD +) Limosilactobacillus fermentum MG4231 and MG4244 in a 1:1 ratio. 
of the HFD group produced significantly high levels of hepatic steatosis and inflammatory cell filtration (Figure 3C). However, MG4244 (3.6 \pm 1.5$)$ and the combination $(3.4 \pm 1.0)$ groups significantly attenuated histopathological changes in liver tissue compared to the HFD $(7.0 \pm 1.4)$ group. The TG content of the liver increased significantly in the HFD group ( $16.72 \pm 0.76 \mathrm{mg} / \mathrm{g})$ compared to the NC group $(7.29 \pm 0.62 \mathrm{mg} / \mathrm{g})$, while MG4231 $(13.24 \pm 1.04 \mathrm{mg} / \mathrm{g})$, MG4244 $(13.28 \pm 1.05 \mathrm{mg} / \mathrm{g})$, and combination $(9.47 \pm 0.77$ $\mathrm{mg} / \mathrm{g}$ ) showed significantly lower levels compared to the HFD group (Figure 3D).

In addition, the diameter of adipocytes in epididymal adipose tissue significantly increased in the HFD group $(106.5 \pm 6.78 \mu \mathrm{m})$ compared to the ND group $(48.46 \pm 5.23$ $\mu \mathrm{m})$ (Figure 3B). However, it was found to decrease the combination-treated group to approximately $91.09 \pm 10.41$ $\mu \mathrm{m}$, considerably smaller than that of the HFD group (Figure 3E).

\section{Effect of Limosilactobacillus fermentum combination on lipogenic protein expression}

The effect of $L$. fermentum strain administration on the expression of obesity-related target proteins in HFDinduced obese mice was confirmed by western blotting analysis. In epididymal adipose tissue (Figure 4), lipogenic protein expression of PPARy, FAS, aP2, and LPL in the HFD group increased significantly by more than three times in the same manner as mRNA expression, compared

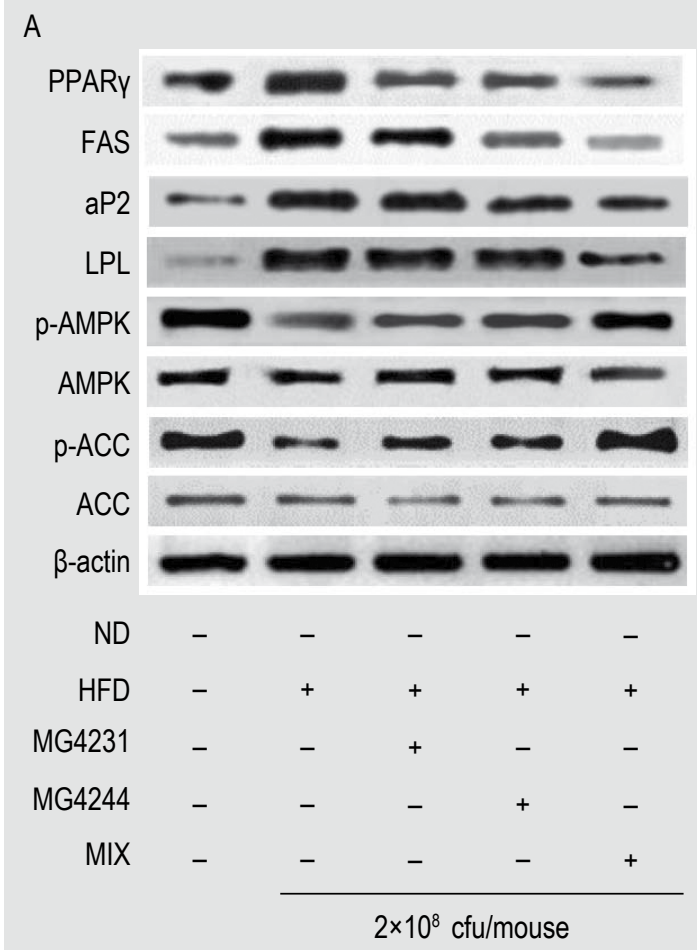

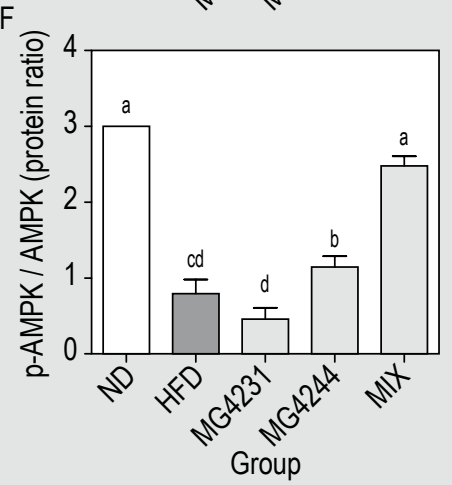
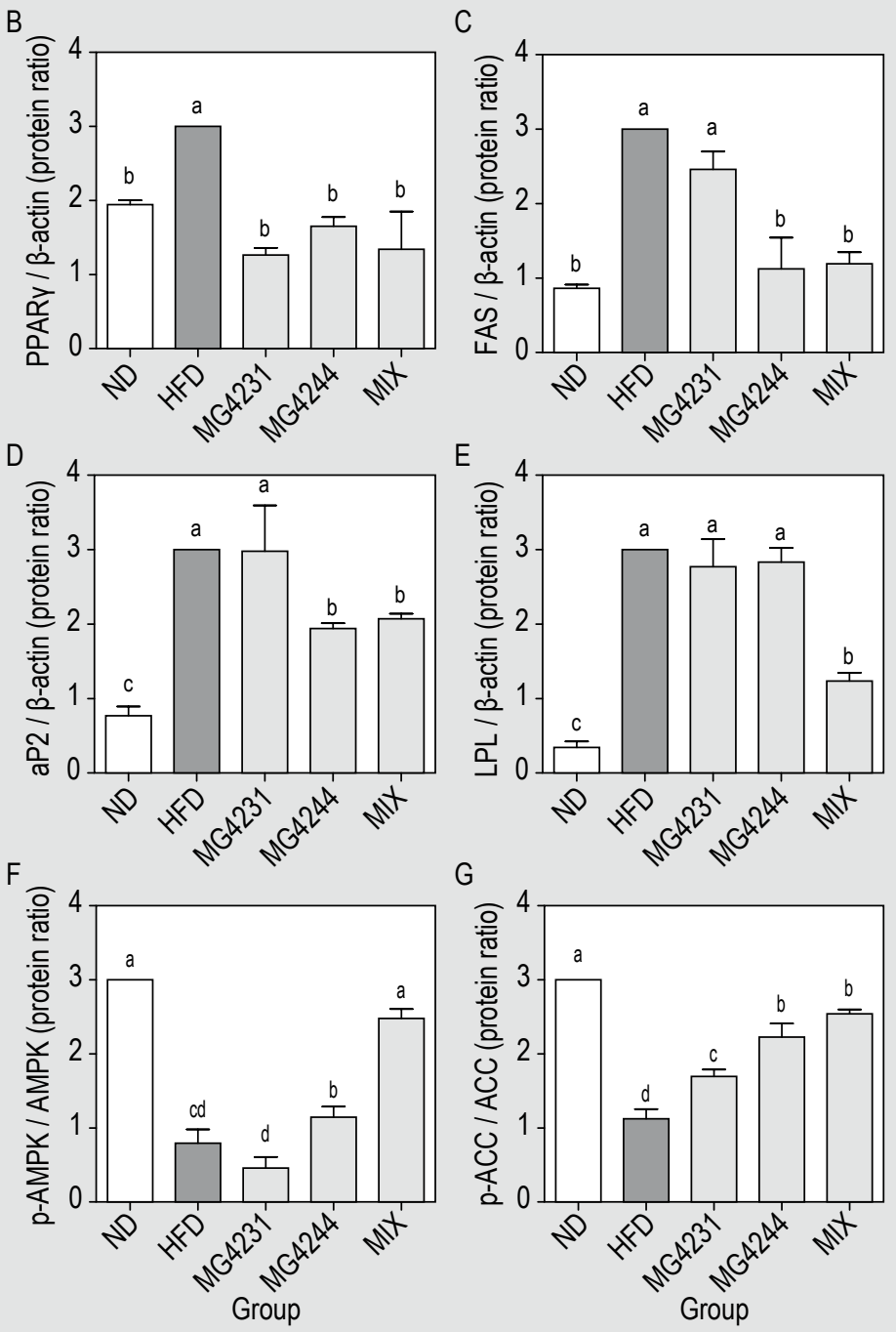

Figure 4. Effect of Limosilactobacillus fermentum strains on lipogenic protein expression in epididymal adipose tissue of HFDinduced obese mice. (A) Western blot image and relative protein levels of (B) PPARY, (C) FAS, (D) aP2, (E) LPL, (F) p-AMPK/ AMPK, and (G) p-ACC/ACC. Results are presented as the mean \pm standard deviation $(n=6)$. Different letters indicate significant differences between means at $P<0.05$. ND = normal-diet fed; HFD = high-fat-diet fed; MIX: (HFD +) Limosilactobacillus fermentum MG4231 and MG4244 in a 1:1 ratio. 
to that in the ND group. In contrast, the expression of PPAR $\gamma$, FAS and aP2 was significantly lower in the MG4244 and combination-treated groups than in the HFD group. HFD feeding markedly reduced AMPK phosphorylation approximately three-fold compared to that in the ND group, whereas the opposite effect was observed in the combination-treated group. Expression of p-ACC, a downstream target protein of AMPK, also significantly decreased in the HFD group compared that in the ND group and increased dramatically in the MG4244 and combination-treated groups. In liver tissue (Figure 5), SREBP-1c significantly increased three-fold in the HFD group compared to that in the ND group but significantly decreased in all L. fermentum strain-treated groups. FAS expression was also reduced considerably in the combination-treated group by approximately $30 \%$ compared to that in the HFD group. In addition, phosphorylated proteins of AMPK and ACC in liver tissue significantly increased in all L. fermentum strain-treated groups compared to the HFD group, showing the same results as the expression in epididymal adipose tissue. Thus, the mechanism of inhibition of fat accumulation in the liver tissue with the administration of $L$. fermentum strains was confirmed.

\section{Discussion}

Obesity is caused by an imbalance between the over-storage of energy and consumption (Ghosh and Bouchard, 2017). An increase in tissue weight manifests as fat accumulation in tissues. HFD-induced rodent obesity models are widely used to study the mechanism of interaction for obesity (Wang and Liao, 2012). Long-term HFD increases the influx of fat into the blood, leading to fat accumulation in the liver and adipose tissue (White et al., 2013). Several studies have reported that probiotics administration affects intestinal hormones that regulate appetite and satiety, reducing energy intake and weight gain in HFD-induced obese mice (Cerdó et al., 2019; Shabana et al., 2018). In this study, the weight gain of mice due to HFD feeding decreased the most by $28.9 \%$ in the combination-treated group, which mixed the two LAB strains (Figure 1A). Epididymal and subcutaneous tissue weights were also reduced by 22 and $58 \%$, respectively, in the combination-treated group (Table 1). These results demonstrated that weight gain in the liver and adipose tissue might be owing fat accumulation in tissue caused by the HFD, and the combination-treated group effectively inhibited body fat accumulation, including in the liver and adipose tissues.
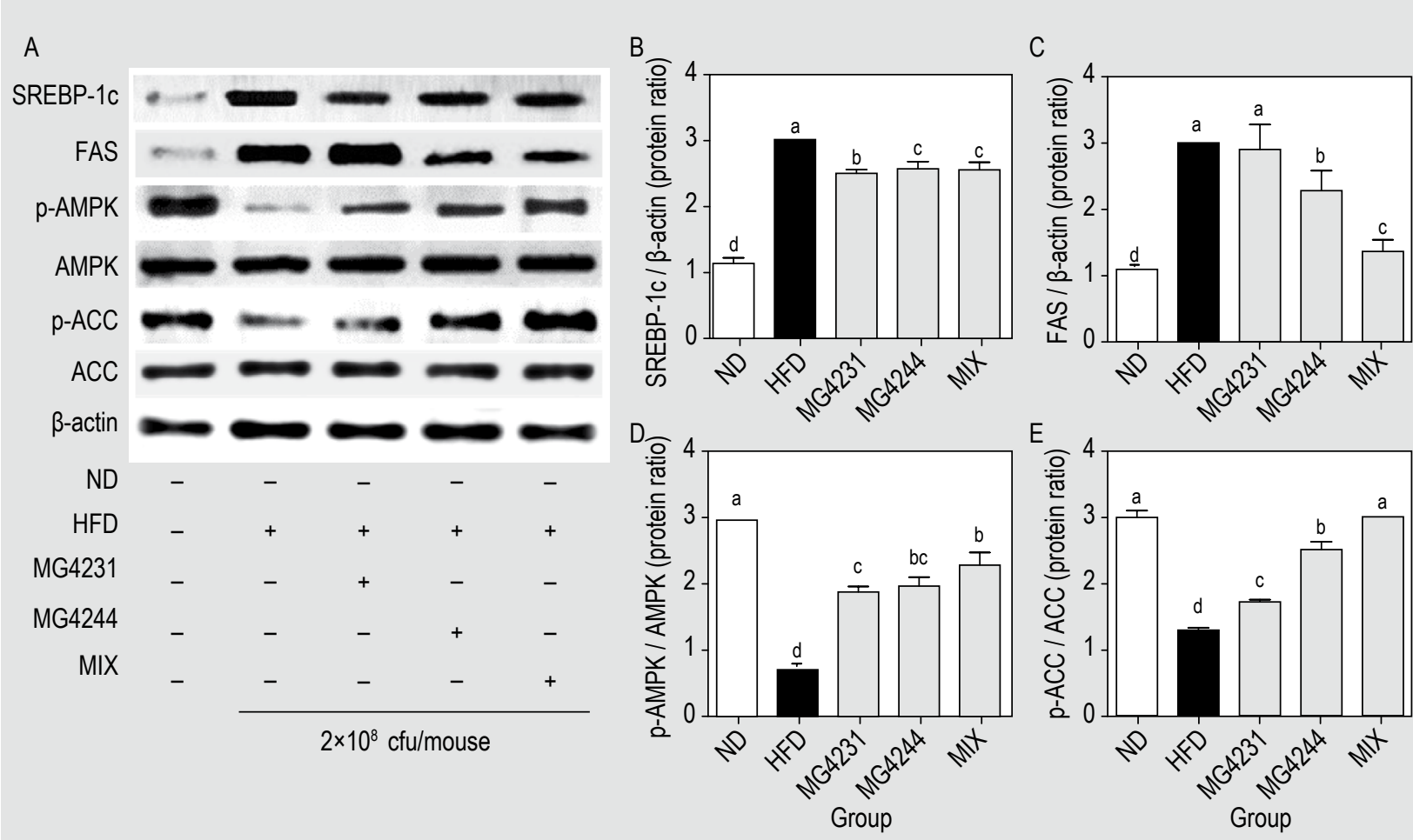

Figure 5. Effect of Limosilactobacillus fermentum strains on the expression of the protein associated with lipid metabolism in the liver tissue of HFD-induced obese mice. (A) Western blot image and relative protein levels of (B) SREBP-1c, (C) FAS, (D) p-AMPKI $A M P K$, and $(E) p-A C C / A C C$. Results are presented as the mean \pm standard deviation $(n=6)$. Different letters indicate significant differences between means at $P<0.05$. ND = normal-diet fed; HFD = high-fat-diet fed; MIX: $(H F D+)$ Limosilactobacillus fermentum MG4231 and MG4244 in a 1:1 ratio. 
Obesity is a leading cause of insulin resistance. Maintaining normal glucose tolerance requires a balance between insulin secretion and function (Ferrannini et al., 1997). In our study, fasting blood glucose level at time 0 was higher in the HFD group than in the LAB intake and ND groups. After glucose administration, the peak blood glucose levels were equal, except in the ND group. However, we observed that the blood glucose level dropped rapidly in the combination-treated group. In a previous study, Williams et al. (2014) reported a rapid increase in OGT between 12 and 16 weeks of HFD induction. L. plantarum MTCC5690 and L. fermentum MTCC5689 improved glucose homeostasis in HFD-induced obese mice by regulating energy metabolism and fatty acid oxidation (Balakumar et al., 2018). L. plantarum and L. fermentum improved glucose tolerance and alleviated hepatic insulin resistance in HFD-fed streptozotocin-induced diabetic mice (Chen et al., 2018). Therefore, these results indicate that administration of the combination may contribute to improving glucose tolerance in HFD-fed mice.

Dyslipidaemia is often accompanied by abnormalities in fat metabolism and glycolysis (Franssen et al., 2011). In obesityinduced mice, probiotics administration allows cholesterollowering factors, such as hydroxy methyl-glutaric acid and orotic acid, to decompose cholesterol, thereby providing a preventive effect against hypercholesterolemia (Ooi and Liong, 2010). Salaj et al. (2013) confirmed the reducing effect of total cholesterol and very low-density lipoprotein in serum by administering two L. plantarum strains to rat with HFD-induced obesity. Park et al. (2014) reported that $L$. plantarum LG42 significantly reduced TG without lowering serum cholesterol levels. In this study, administration of the combination significantly reduced serum ALT, TC, and LDL levels. However, HDL-C, TG, and glucose levels were not statistically different from those in the HFD group. Similar to our findings, Bosch et al. (2013) reported that the $L$. plantarum mixture had a serum cholesterol-lowering effect but did not affect TG, HDL, or glucose levels in obese mice.

Leptin and adiponectin are adipokines secreted primarily from adipocytes and play an important role in regulating energy homeostasis (Rosenbaum et al., 1997). Recent studies have shown that the hormone leptin is produced in proportion to the amount of adipose tissue and is closely related to obesity (Jequier, 2002; Kang et al., 2013). In addition, when leptin increases, obesity is caused by changes in the gut microflora composition, with an increase in Firmicutes and decrease in Bacteroidetes (Heiss and Olofsson, 2018). Takemura et al. (2010) also reported that L. plantarum strain No. 14 reduced TC and leptin levels in the serum of HFD-fed mice. In this study, the leptin level significantly elevated by HFD feeding decreased when $L$. fermentum was administered alone or in combination, whereas adiponectin levels increased in the MG4244 and combination-treated groups. Adiponectin in fat, muscle, and liver tissue improves insulin sensitivity and maintains glucose homeostasis (Achari and Jain, 2017). In obesity, insulin sensitivity decreases, and adiponectin synthesis in adipose tissue is known to decrease (Barnea et al., 2006). In this study, we found that adiponectin levels recovered to the chow group level with MG4244 and combination therapy. Similar to our results, Wu et al. (2015) reported that L. plantarum K21 treatment recovered adiponectin levels of the chow group in HFD-induced obese mice. Wang et al. (2020) showed that five Lactobacillus strains including $L$. fermentum NCU0413 improved obesity in HFD-induced obese mice by relieving lipid metabolism and controlling leptin and adiponectin. Therefore, these results show that the administration of the combination effectively improved the serum biochemical changes caused by HFD.

In obesity, adipocyte hypertrophy is enlarged, and tissues have a low level of inflammatory environmental conditions. Lipids accumulate gradually in the form of TG in the liver caused of persistent oversupply and impaired fatty acid metabolism. Hepatic steatosis and inflammation are characteristics of NAFLD that can exacerbate lipidmediated damage (Sarwar et al., 2018). Recent studies have reported that probiotics improve insulin resistance and hepatic steatosis in HFD-fed mice (Jang et al., 2019; Xie and Halegoua-DeMarzio, 2019). In this study, a HFD induced fat accumulation in the liver, and it was confirmed that the strain alone or in combination improved lipid accumulation in the liver. Administration of the combination suppressed hepatic steatosis and reduced TG levels in the liver tissue by $43 \%$. It also reduced the adipocyte size by $14 \%$ (Figure 3D). A previous study showed that Lactobacillus acidophilus NS1 significantly reduced hepatic lipid accumulation (Park et al., 2018). Zhu et al. (2019) reported that L. fermentum CQPC05 reduced obesity-related pathological changes and hepatocyte enlargement in the liver tissue of HFDobese mice. Specific LAB administration inhibits adipocyte hypertrophy by reducing leptin in the serum (Caricilli and Saad, 2014). Therefore, suppressing the hypertrophy of adipocytes in the L. fermentum administered group may be owing to a reduction in serum leptin levels. These results demonstrate that the combination is involved in inhibiting fat accumulation in the liver and adipose tissues and attenuating FLD progression.

Obesity is a complex disease caused by various genetic variations and interactions with the environment (Fujioka, 2002). Excessive absorption of saturated fats increases TG storage and hepatitis, indirectly increasing insulin resistance and the number of lipid-related genes (Li et al., 2019). Lipogenesis is regulated by transcription factors, such as PPARs and SREBPs. In adipose tissue, most PPAR $\gamma$-target genes in adipose tissue are directly related to lipogenic pathways, such as aP2, FAS, and LPL, which are highly detectable in the liver or adipose tissue and whose activity is known to be sensitive to nutritional status (Moseti et 
al., 2016). AMPK plays a critical role in lipid homeostasis as a regulator of fat production and fatty acid oxidation in the adipose tissue, liver, and skeletal muscles through the modulation of transcription factors (Ruderman et al., 2013). Kim et al. (2013) reported that L. rhamnosus GG reduced lipid accumulation by downstream activation of AMPK in mice fed a HFD for 13 weeks. In this study, it was confirmed that the inhibition of fat accumulation in epididymal adipose tissue by the administration of the combination was because of the upregulation of ACC and AMPK. In a previous in vitro study using 3T3-L1 cells, we demonstrated that the anti-obesity activity of MG4231 and MG4244 is due to the inhibition of adipogenesis-specific genes by regulating AMPK phosphorylation. In this study, these mechanisms confirmed the same results in a HFDinduced obesity mouse model.

SREBP-1c is a cytoplasmic transcription factor primarily activated by insulin and regulates the expression of genes required for cholesterol, fatty acid, TG, and phospholipid synthesis. SREBP-1c stimulates FAS, an enzyme involved in liver fat synthesis, leading to fatty acids and TG synthesis. Thus, the overexpression of SREBP-1c, FAS, and ACC1, major transcription factors in lipid synthesis, is associated with obesity and chronic fatty liver development (Postic and Girard, 2008). In a previous study, the combination of L. rhamnosus GG with L. sakei NR28 reduced ACC and FAS in the liver of mice fed a HFD (Ji et al., 2012). Choi et al. (2020) reported that administration of L. plantarum LMT1-48 reduced liver weight and TG concurrently with the downregulation of lipogenic genes PPAR $\gamma, \mathrm{HSL}, \mathrm{SCD}-$ 1 , and FAT/CD36 in the liver. Similar to the results of a previous study, the administration of the combination in this study inhibited the expression of SREBP-1c, FAS, and ACC by increasing the phosphorylation of ACC and AMPK in liver tissue. These results demonstrate that the antiobesity activity of the combination of MG4231 and MG4244 is achieved by upregulating the protein phosphorylation of AMPK/ACC, thereby downregulating the expression of adipogenic proteins. Therefore, it is suggested that the administration of the combination of L. fermentum MG4231 and MG4244 may have a beneficial effect in improving fat accumulation in the liver and adipose tissue of mice with HFD-induced obesity.

\section{Conclusions}

We found that the administration of the combination of MG4231 and MG4244 in HFD-fed obese C57BL/6J mice further reduced weight gain, fat accumulation, inflammation, and adipocyte expansion in the liver and adipose tissues compared to individual administration of the strains. The combination treatment group also showed improved serum profiles, including TC, LDL-c, and leptin. The anti-obesity activity of the combination was found to modulate lipid metabolism and fat accumulation by upregulating the phosphorylation of the AMPK/ACC signalling pathway in the liver and adipose tissue. Therefore, the combination of L. fermentum MG4231 and MG4244 may be a potential probiotic candidate for the prevention of obesity.

\section{Acknowledgements}

This research was conducted with support from the project for Collabo R\&D between Industry, Academy, and Research Institute funded by Korea Ministry of SMEs and Startups in 2019 (Project No. S2717946).

\section{Conflicts of interests}

No potential conflict of interest was reported by the authors.

\section{References}

Abenavoli, L., Scarpellini, E., Colica, C., Boccuto, L., Salehi, B., Sharifi-Rad, J., Aiello, V., Romano, B., Lorenzo, A.D., Angelo, A. and Capasso, I.R., 2019. Gut microbiota and obesity: a role for probiotics. Nutrients 11: 2690. https://doi.org/10.3390/nu11112690

Achari, A.E. and Jain, S.K., 2017. Adiponectin a therapeutic target for obesity, diabetes, and endothelial dysfunction. International Journal of Molecular Sciences 18: 1321. https://doi.org/10.3390/ ijms 18061321

Andrikopoulos, S., Blair, A.R., Deluca, N., Fam, B.C. and Proietto, J., 2008. Evaluating the glucose tolerance test in mice. American Journal of Physiology - Endocrinology and Metabolism 295: E1323-E1332. https://doi.org/10.1152/ajpendo.90617.2008

Balakumar,M., Prabhu, D., Sathishkumar, C., Prabu, P, Rokana, N., Kumar, R., Raghavan, S., Soundarajan, A, Grover, S, Batish, V., Mohan, V. and Balasubramanyam, M., 2018. Improvement in glucose tolerance and insulin sensitivity by probiotic strains of Indian gut origin in high-fat diet-fed C57BL/6J mice. European Journal of Clinical Nutrition 57: 279-295. http://doi: 10.1007/s00394-0161317-7

Barnea, M., Shamay, A., Stark, A.H. and Madar, Z., 2006. A high-fat diet has a tissue-specific effect on adiponectin and related enzyme expression. Obesity 14: 2145-2153.

Bosch, M., Fuentes, M.C., Audivert, S., Bonachera, M.A., Peiró, S. and Cuñé, J., 2013. Lactobacillus plantarum CECT 7527, 7528 and 7529: probiotic candidates to reduce cholesterol levels. Journal of the Science of Food Agriculture 94: 803-809. https://doi.org/10.1002/ jsfa.6467

Bubnov, R.V., Babenko, L.V., Lazarenko, L.M., Mokrozub, V.V. and Spivak, M.Y., 2018. Specific properties of probiotic strains: relevance and benefits for the host. EPMA Journal 9: 205-223. https://doi. org/10.1007/s13167-018-0132-z

Cai, H., Wen, Z., Li, X., Meng, K. and Yang, P., 2020. Lactobacillus plantarum FRT10 alleviated high-fat diet-induced obesity in mice through regulating the PPAR $\alpha$ signal pathway and gut microbiota. Applied Microbiology and Biotechnology 104: 5959-5972. https:// doi.org/10.1007/s00253-020-10620-0 
Caricilli, A.M. and Saad, M.J., 2014. Gut microbiota composition and its effects on obesity and insulin resistance. Current Opinion in Clinical Nutrition and Metabolic Care 17: 312-318. https://doi. org/10.1097/MCO.0000000000000067

Cerdó, T., García-Santos, J.A., Bermúdez, M.G. and Campoy, C., 2019. The role of probiotics and prebiotics in the prevention and treatment of obesity. Nutrient 11: 635. https://doi.org/10.3390/nu11030635

Chen, D., Yang, Z., Chen, X., Huang, Y., Yin, B., Guo, F., Zhao, H., Zhao, T., Qu, H., Huang, J., Wu, Y. and Gu, R., 2014. The effect of Lactobacillus rhamnosus hsryfm 1301 on the intestinal microbiota of a hyperlipidemic rat model. BMC Complementary and Alternative Medicine 10: 386. http://doi: 10.1186/1472-6882-14-386

Chen, X., Tan, F., Yi, R., Mu, J., Zhao, X. and Yang, Z., 2018. Effect of Lactobacillus on mice with diabetes induced by hight-fat diet with streptozotocin (STZ). Applied Sciences 8: 1249. https://doi. org/10.3390/app8081249

Choi, C.H. and Chang, S.K., 2015. Alteration of gut microbiota and efficacy of probiotics in functional constipation. Journal of Neurogastroenterology and Motility 21: 4-7. https://doi.org/10.5056/ jnm14142

Choi, W.J., Dong, H.J., Jeong, H.U., Jung, H.H., Kim, Y.H. and Kim, T.H., 2019. Antiobesity effects of Lactobacillus plantarum LMT1-48 accompanied by inhibition of Enterobacter cloacae in the intestine of diet-induced obese mice. Journal of Medicinal Food 22: 560-566. https://doi.org/10.1089/jmf.2018.4329

Choi, W.J., Dong, H.J., Jeong, H.U., Ryu, D.W., Song, S.M., Kim, Y.R., Jung, H.H., Kim, T.H. and Kim, Y.H., 2020. Lactobacillus plantarum LMT1-48 exerts anti-obesity effect in high-fat diet-induced obese mice by regulating expression of lipogenic genes. Sciencific Reports 10: 869. https://doi.org/10.1038/s41598-020-57615-5

Ferrannini, E., Natali, A., Bell, P., Cavallo-Perin, P., Lalic, N. and Mingrone, G., 1997. Insulin resistance and hypersecretion in obesity. European group for the study of insulin resistance (EGIR). Journal of Clinical Investigation 100: 1166-1173. https://doi.org/10.1172/ JCI119628

Franssen, R., Monajemi, H., Stroes, E.S.G. and Kastelein, J.J.P., 2011. Obesity and dyslipidemia. Medical Clinics of North America 95: 893-902. https://doi.org/10.1016/j.mcna.2011.06.003

Fujioka, K., 2002. Management of obesity as a chronic disease: nonpharmacologic, pharmacologic, and surgical options. Obesity Research 10: 116S-123S. https://doi.org/10.1038/oby.2002.204.

Ghosh, S. and Bouchard, C., 2017. Convergence between biological, behavioural and genetic determinants of obesity. Nature Reviews Genetics 18: 731-748. https://doi.org/10.1038/nrg.2017.72

Heiss, C.N. and Olofsson, L.E., 2018. Gut microbiota-dependent modulation of energy metabolism. Journal of Innate Immunity 10: 163-171. https://doi.org/10.1159/000481519

Hussain, A., Kwon, M.H., Kim, H.K., Lee, H.S., Cho, J.S. and Lee, Y.I., 2020. Anti-obesity effect of Lactobacillus plantarum LB818 is associated with regulation of gut microbiota in high-fat diet-fed obese mice. Journal of Medicinal Food 23: 750-759. https://doi. org/10.1089/jmf.2019.4627
Jang, H.R., Park, H.J., Kang, D., Chung, H., Nam, M.H., Lee, Y., Park, J.H. and Lee, H.Y., 2019. A protective mechanism of probiotic Lactobacillus against hepatic steatosis via reducing host intestinal fatty acid absorption. Experimental and Molecular Medicine 51: 1-14. https://doi.org/10.1038/s12276-019-0293-4.

Jequier, E., 2002. Leptin signaling, adiposity, and energy balance. Annals of the New York Academy of Sciences 967: 379-388. https://doi. org/10.1111/j.174.9-6632.2002.tb04293.x

Ji, Y.S., Kim, H.N., Park, H.J., Lee, J.E., Yeo, S.Y., Yang, J.S., Park, S.Y., Yoon, H.S., Cho, G.S., Franz, C.M. A.P., Bomba, A., Shin, H.K. and Holzapfel, W.H., 2012. Modulation of the murine microbiome with a concomitant anti-obesity effect by Lactobacillus rhamnosus GG and Lactobacillus sakei NR28. Beneficial Microbes 3: 13-22. https:// doi.org/10.3920/BM2011.0046

Ji, Y.S., Park, S., Chung, Y., Kim, B., Park, H., Huang, E., Jeong, D., Jung, H.Y., Kim, B., Hyun, C.K. and Holzapfel, W.H., 2019. Amelioration of obesity-related biomarkers by Lactobacillus sakei CJLS03 in a high-fat diet-induced obese murine model. Scientific Reports 9: 6821. https://doi.org/10.1038/s41598-019-43092-y

Kang, C.H., Han, S.H., Kim, Y., Paek, N.S. and So, J.S., 2018. In vitro probiotic evaluation of potential antiobesity lactic acid bacteria isolated from human vagina and shellfish. Korean Society for Biotechnology and Bioengineering Journal 33: 161-167. https:// doi.org/10.784.1/ksbbj.2018.33.3.161

Kang, J.H., Yun, S.I., Park, M.H., Park, J.H., Jeong, S.Y. and Park, H.O., 2013. Anti-obesity effect of Lactobacillus gasseri BNR17 in highsucrose diet-induced obese mice. PLoS ONE 8: e54617. https://doi. org/10.1371/journal.pone.0054617

Kim, S., Choi, S.I., Jang, M., Jeong, Y. and Kang, C.H., 2020. Antiadipogenic effect of Lactobacillus fermentum MG4231 and MG4244 through AMPK pathway in 3T3-L1 preadipocytes. Food Science and Biotechnology 29: 1541-1551. https://doi.org/10.1007/s10068020-00819-2

Kim, S.W., Park, K.Y., Kim, B., Kim, E. and Hyun, C.K., 2013. Lactobacillus rhamnosus GG improves insulin sensitivity and reduces adiposity in high-fat diet-fed mice through enhancement of adiponectin production. Biochemical and Biophysical Research Communications 431: 258-263. https://doi.org/10.1016/j. bbrc.2012.12.121

Lee, E., Jung, S.R., Lee, S.Y., Lee, N.K., Paik, H.D. and Lim, S.I., 2018. Lactobacillus plantarum strain Ln4 attenuates diet-induced obesity, insulin resistance, and changes in hepatic mRNA levels associated with glucose and lipid metabolism. Nutrients 19: 643. https://doi. org/10.3390/nu10050643

Ley, R.E., Bäckhed, F., Turnbaugh, P., Lozupone, C.A., Knight, R.D. and Gordon, J.I., 2005. Obesity alters gut microbial ecology. Proceedings of the National Academy of Sciences of the United States of America 102: 11070-11075. https://doi.org/10.1073/pnas.0504978102

Li, H., Liu, F., Lu, J., Shi, J., Guan, J., Yan, F, Li, B. and Huo, G., 2020. Probiotic mixture of Lactobacillus plantarum strains improved lipid metabolism and gut microbiota structure in high fat diet-fed mice. Fronts in Microbiology 26: 512. https://doi.org/10.3389/ fmicb.2020.00512 
Li, T., Yan, H., Geng, Y., Shi, H., Li, H., Wang, S., Wang, Y., Xu, J., Zhao, G. and Lu, X., 2019. Target genes associated with lipid and glucose metabolism in non-alcoholic fatty liver disease. Lipids in Health and Disease 18: 211. https://doi.org/10.1186/s12944-019-1154-9

Liang, W., Menke, A.L., Driessen, A., Koek, G.H., Lindeman, J.H., Stoop, R., Havekes, L.M., Kleemann, R. and Van den Hoek, A.M., 2014. Establishment of a general NAFLD scoring system for rodent models and comparison to human liver pathology. PLoS ONE 9: e115922. https://doi.org/10.1371/journal.pone.0115922

Moseti, D., Regassa, A. and Kim, W.K., 2016. Molecular regulation of adipogenesis and potential anti-adipogenic bioactive molecules. International Journal of Molecular Science 17: 124. https://doi. org/10.3390/ijms17010124

National Research Council (NRC), 1996. Guide for the care and use of laboratory animals. National Research Council, Institute for Laboratory Animal Research. National Academies Press, Washington, DC, USA.

Nguyen, T.H., Kim, Y., Kim, J.S., Jeong, Y., Park, H.M., Kim, J.W., Kim, J.E., Kim, H., Paek, N.S. and Kang, C.H., 2020. Evaluating the cryoprotective encapsulation of the lactic acid bacteria in simulated gastrointestinal conditions. Biotechnology and Bioprocess Engineering 25: 287-292. https://doi.org/10.1007/s12257-019-0406-x

Nuttall, F.Q., 2015. Body mass index: obesity, BMI, and health: a critical review. Nutrition Today 50: 117-128. https://doi.org/10.1097/ NT.0000000000000092

Ooi, L.G. and Liong, M.T., 2010. Cholesterol-lowering effects of probiotics and prebiotics: a review of in vivo and in vitro findings. International Journal of Molecular Science 11: 2499-2522. https:// doi.org/10.3390/ijms11062499

Paley, C.A. and Johnson, M.I., 2018. Abdominal obesity and metabolic syndrome: exercise as medicine? BMC Sports Science, Medicine and Rehabilitation 10: 7. https://doi.org/10.1186/s13102-018-0097-1

Park, J.E., Oh, S.H. and Cha, Y.S., 2014. Lactobacillus plantarum LG42 isolated from gajami sik-hae decreases body and fat pad weights in diet-induced obese mice. Journal of Applied Microbiology 116: 145-156. https://doi.org/10.1111/jam.12354

Park, S.S., Lee, Y.J., Song, S., Kim, B., Kang, H., Oh, S. and Kim, E., 2018. Lactobacillus acidophilus NS1 attenuates diet-induced obesity and fatty liver. Journal of Endocrinology 237: 87-100. https://doi. org/10.1530/JOE-17-0592

Postic, C. and Girard, J., 2008. Contribution of de novo fatty acid synthesis to hepatic steatosis and insulin resistance: lessons from genetically engineered mice. Journal of Clinical Investigation 118: 829-838. https://doi.org/10.1172/JCI34275

Rosenbaum, M., Nicolson, M., Hirsch, J., Murphy, E., Chu, F. and Leibel, R.L., 1997. Effect of weight change on plasma leptin concentrations and energy expenditure. The Journal of Clinical Endocrinology and Metabolism 82: 3647-3654. https://doi.org/10.1210/jcem.82.11.4390

Ruderman, N.B., Carling, D., Prentki, M. and Cacicedo, J.M., 2013. AMPK, insulin resistance, and the metabolic syndrome. Journal of Clinical Investigation 123: 2764-2772. https://doi.org/10.1172/ JCI67227

Salaj, R., Štofilová, J., Šoltesová, A., Hertelyová, Z., Hijová, E., Bertková, I., Strojný, L., Kružliak, P. and Bomba, A., 2013. The effects of two Lactobacillus plantarum strains on rat lipid metabolism receiving a high fat diet. Scientific World Journal 2013: 135142.
Sarwar, R., Pierce, N. and Koppe, S., 2018. Obesity and nonalcoholic fatty liver disease: current perspectives. Diabetes Metabolic Syndrome and Obesity 11: 533-542. https://doi.org/10.2147/DMSO. S146339

Sethunath, D., Morusu, S., Tuceryan, M., Cummings, O.W., Zhang, H., Yin, X.M., Vanderbeck, S., Chalasani, N. and Gawrieh, S., 2018. Automated assessment of steatosis in murine fatty liver. PLoS ONE 13: e0197242. https://doi.org/10.1371/journal.pone.0197242

Shabana, Shahid, S.U. and Irfan, U., 2018. The gut microbiota and its potential role in obesity. Future Microbiology 13: 589-603. https:// doi.org/10.2217/fmb-2017-0179

Srivastava, N.K., Pradhan, S., Mittal, B., Kumar, R. and Gowda, G.N., 2006. An improved, single step standardized method of lipid extraction from human skeletal muscle tissue. Analytical Letters 39: 297-315. https://doi.org/10.1080/00032710500477001

Takemura, N., Okubo, T. and Sonoyama, K., 2010. Lactobacillus plantarum strain No. 14 reduces adipocyte size in mice fed high-fat diet. Experimental Biology and Medicine 235: 849-856. https://doi. org/10.1258/ebm.2010.009377

Tarrah, A., D, J., Rossi, R.C., Da Silva Duarte, V., Ziegler, D.R., Corich, V. and Giacomini, A., 2018. In vitro probiotic potential and anticancer activity of newly isolated folate-producing Streptococcus thermophilus strains. Frontier in Microbiology 9: 2214. https://doi. org/10.3389/fmicb.2018.02214

Trayhurn, P. and Beattie J.H., 2001. Physiological role of adipose tissue: white adipose tissue as an endocrine and secretory organ. Proceedings of the Nutrition Society 60: 329-339. https://doi. org/10.1079/pns200194

Walker, J.M., 1994. The bicinchoninic acid (BCA) assay for protein quantitation. Methods in Molecular Biology 32: 5-8. https://doi: 10.1385/0-89603-268-X:5

Wang, C.Y. and Liao, J.K., 2012. A mouse model of diet-induced obesity and insulin resistance. Methods in Molecular Biology 821: 421-433. https://doi.org/10.1007/978-1-61779-430-8_27

Wang, M., Zhang, B., Hua, J., Nie, S., Xiong, T. and Xie, M., 2020. Intervention of five strains of Lactobacillus on obesity in mice induced by high-fat diet. Journal of Functional Foods 72: 104078. https://doi.org/10.1016/j.jff.2020.104078

White, P.A., Cercato, L.M., Araújo, J.M., Souza, L.A., Soares, A.F., Barbosa, A.P., Neto, J.M., Marçal, A.C., Machado, U.F., Camargo, E.A., Santos, M.R. and Brito, L.C., 2013. Model of high-fat dietinduced obesity associated to insulin resistance and glucose intolerance. Arquivos Brasileiros de Endocrinologia and Metabologia 57: 339-345. https://doi.org/10.1590/s0004-27302013000500002

Wilkins, T. and Sequoia, J., 2017. Probiotics for gastrointestinal conditions: a summary of the evidence. American Family Physician 96: $170-178$

Williams, L.M., Campbell, F.M., Drew, J.E., Koch, C., Hoggard, N., Rees, W.D. Kamolrat, T., Ngo, H.T., Steffensen, I., Gray, S.R. and Tups, A., 2014. The development of diet-induced obesity and glucose intolerance in C57BL/6 mice on a high-fat diet consists of distinct phases. PLoS ONE 9: e106159. https://doi.org/10.1371/journal. pone.0106159

World Health Organization (WHO), 2020. Obesity and overweight. WHO, Geneva, Switzerland. Available at: https://www.who.int/ news-room/fact-sheets/detail/obesity-and-overweight 
Wu, E.C., Weng, W.L., Lai, W.L., Tsai, H.P., Liu, W.H., Lee, M.W. and Tsai, Y.C., 2015. Effect of Lactobacillus plantarum strain K21 on high-fat diet-fed obese mice. Evidence-Based Complementary and Alternative Medicine 2015: 391767. https:// doi.org/10.1155/2015/391767

Xie, C. and Halegoua-DeMarzio, D., 2019. Role of probiotics in nonalcoholic fatty liver disease: does gut microbiota matter? Nutrients 11: 2837. https://doi.org/10.3390/nu11112837
Zhang, Q., Wu, Y. and Fei, X., 2015. Effect of probiotics on glucose metabolism in patients with type 2 diabetes mellitus: a meta-analysis of randomized controlled trials. Medicina 52: 28-34. https://doi. org/10.1016/j.medici.2015.11.008

Zhu, K., Tan, F., Mu, J., Yi, R., Zhou, X. and Zhao, X., 2019. Antiobesity effects of Lactobacillus fermentum CQPC05 isolated from Sichuan pickle in high-fat diet-induced obese mice through PPAR- $\alpha$ signaling pathway. Microorganisms 7: 194. https://doi.org/10.3390/ microorganisms7070194 
